\title{
The impact of the dollar rate on fluctuations of ruble liquidity in Russia
}

\author{
S. M. Borodachev \\ Ural Federal University named after the first President of Russia Boris Yeltsin, \\ Ekaterinburg, Russia \\ s.m.borodachev@gmail.com
}

\begin{abstract}
The influence of both the absolute values of the dollar/ruble exchange rate (rate) and its changes per day on the balance of the Bank of Russia operations for ruble liquidity provision and absorption (saldo) was investigated. Daily data were used from January 2015 to April 2018. It was found that the change in the rate 6 days ago is the cause (according to Granger) of the saldo value. For the saldo dynamics, an oscillatory model with an external force - a change in the rate - is proposed. Using the Kalman filter, the model parameters were estimated and saldo forecasted. Found period of self-oscillation is 4.218 days and attenuation of the amplitude for a day in 2.179 times. The rate growth of 1 RUB, after 6 days, causes saldo increase of approximately 20 billion rubles. In fact, the changes in rate cause the variability of the saldo not more than for found coefficient of determination (26.7\%), but the "change in the rate-liquidity saldo" system during the crisis-free period has a high "Q-factor," and changes in the rate, repeated with a period close to self-one, can cause large-amplitude fluctuations in saldo.
\end{abstract}

Keywords: monetary liquidity, exchange rates, Granger causality, Kalman filter, adequacy error

AMS Classification 2010: 93E12, 91B84

\section{Introduction}

In Russia, there is still a great distrust in the national currency, especially when external unfavorable factors arise. The public and banks at this time buy currency either from a sense of survival, or with speculative purposes. The ruble liquidity required for this is borrowed from the Central Bank on various terms, in particular in the form of repo transactions and currency swaps. It is important to find out whether the changes in the dollar rate that have already occurred are the cause of liquidity changes or vice versa, the speculative play of banks in the foreign exchange market is driving the dollar rate? Then you can build an adequate model of this financial subsystem and work with it using a Kalman filter (KF), widely applied in economic research (see, for example, [1-3]). Another aspect of the relationship of liquidity (the difference in bond yields in two countries) and the exchange rate of their currencies was considered in [4].

\section{Data and causality}

The balance of the Bank of Russia's operations with the banking sector $M^{t}$ (billion rubles) for the provision and absorption of ruble liquidity (hereinafter referred to as saldo) was selected as an indicator of fluctuations in the money supply. This indicator takes into account the operations carried out in previous days, with the terms of execution on the current day $t$ (data at the beginning of the day) and does not take into account the operations, which will be carried out during the current day with the terms of execution on the same day. The negative sign of the balance characterizes the absorption of funds by the Bank of Russia, the positive sign is the provision of liquidity to the banking sector. Data from 13.01.2015 to 28.04.2018 $(t=0, \ldots, 1201)$ were taken from the website of the Central Bank of Russia (http://www.cbr.ru/hd_base/saldo/), to them missed dates filled with interpolated values have been added. Data on the official dollar/ruble rate $S^{t}$ (RUB for 1 USD) (http://www.cbr.ru/currency_base/dynamics/) were processed the same way. See Table 1.

Table 1. Saldo of provision and absorption liquidity and
dollar/ruble rate
\begin{tabular}{|l|l|l|l|l|l|l|l|l|l|}
\hline$t$ & 0 & 1 & 2 & 3 & 4 & 5 & $\ldots$ & 1200 & 1201 \\
\hline$M^{t}$ & 359.3 & 353.7 & 500.4 & 341 & 323.4 & 305.7 & $\ldots$ & 145.8 & 165.7 \\
\hline$S^{t}$ & 62.74 & 64.84 & 66.10 & 64.83 & 65.17 & 65.11 & $\ldots$ & 62.60 & 62.72 \\
\hline
\end{tabular}

\subsection{Absolute values of the dollar exchange rate}

Consider the relationship between absolute values of the exchange rate and saldo. Because it is possible the 
existence of the lag (delay) $L$ in one value's effect on another, we first compute the estimate of the cross-correlation function $\hat{\rho}\left(S^{t-L}, M^{t}\right)$ when the rate precedes the saldo by $L$ days. See Fig. 1.

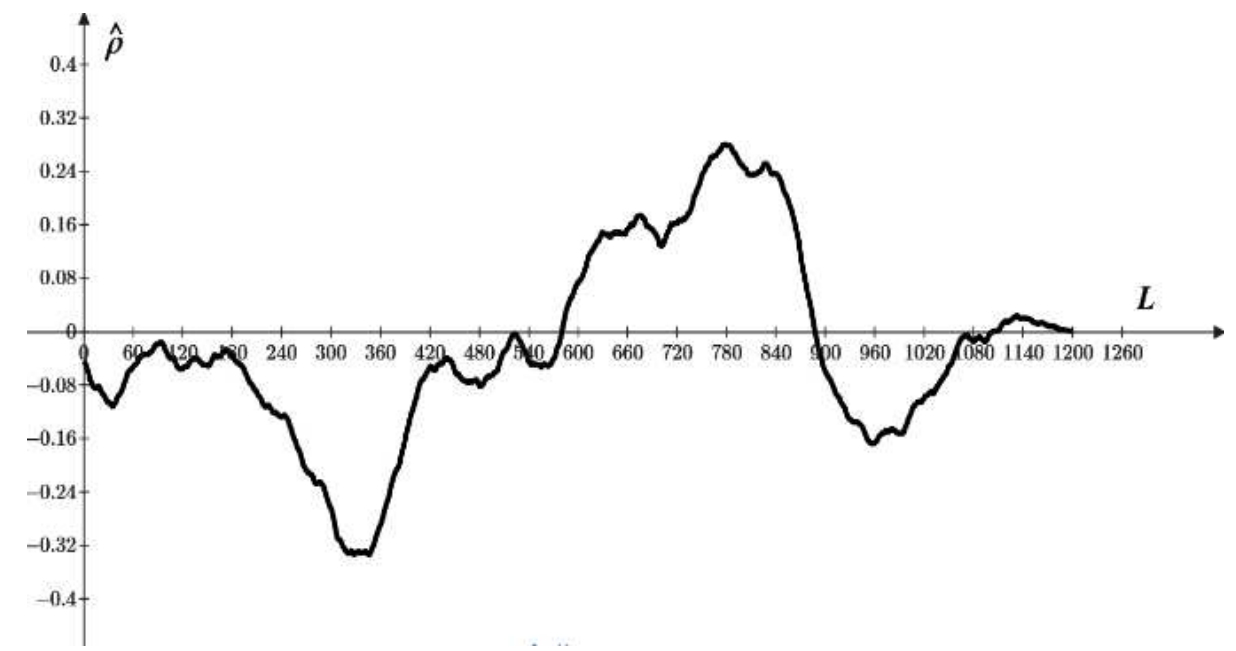

Figure 1. Estimates of rate/saldo correlations for various delays $L$ of saldo.

The most significant value of the correlation coefficient $(-0.383)$ with the lag $L=346$, the significance level ( $s l$ ) according to the $t$-criterion is equal to 0.00000 .... A similar but already positive correlation is observed with a lag of about 2 years. We can suggest the following mechanism for the formation of such a rate effect on liquidity. If about a year ago, the dollar rate was low, then the public and commercial banks assuming that it now also will be low borrowed ruble loans to buy the dollars. Saldo is positive. A year later, these loans have to be returned - the saldo is negative.

If we consider saldo leading the rate, then as much significant correlations will not be found.

\subsection{Changes in the dollar exchange rate}

The main purpose of this work is to study the short-term effects of changes in the rate on the saldo and the forecasts for saldo day ahead. Calculate the rate changes in relation to the previous day $\Delta S^{t}=S^{t}-S^{t-1}$ and first estimate the cross-correlation function $\hat{\rho}\left(\Delta S^{t-L}, M^{t}\right)$ when the rate change precedes the saldo by $L$ day. See Fig. 2.

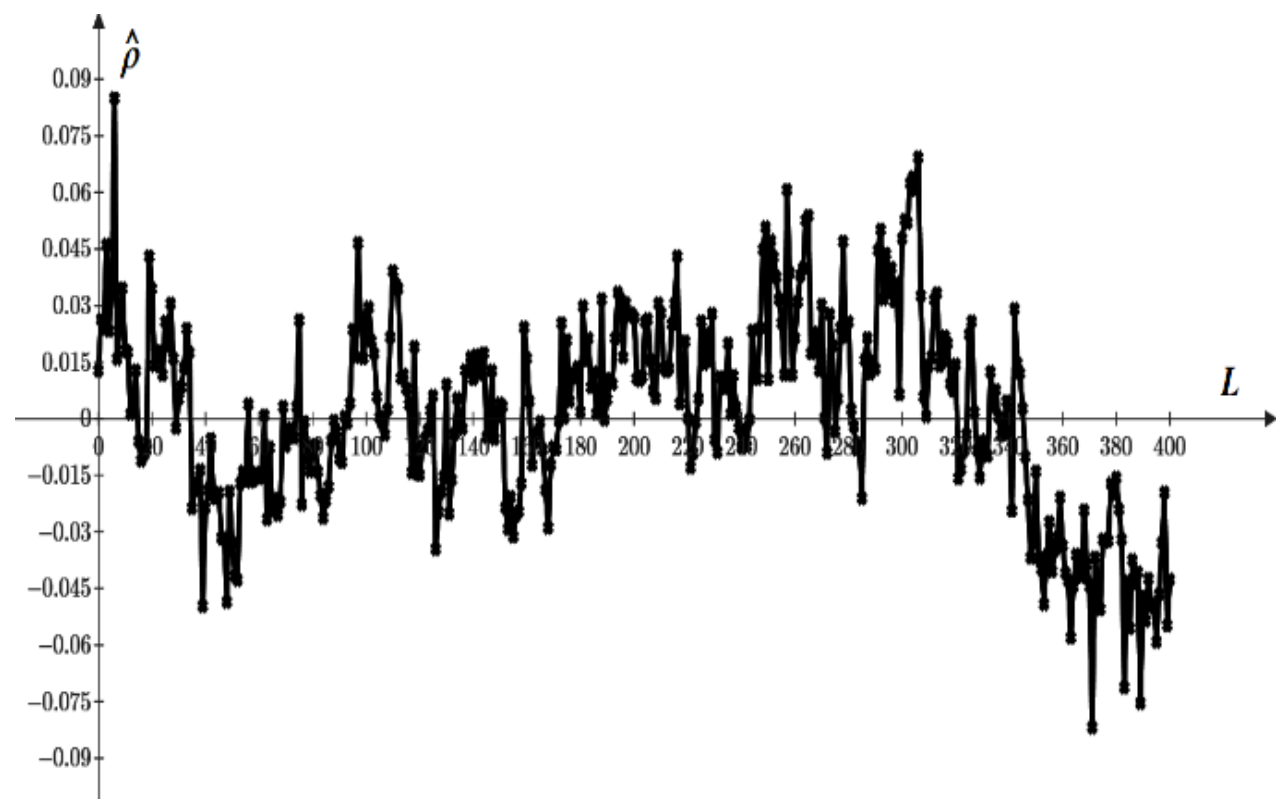

Figure 2. Estimates of and change of rate/saldo correlations for various delays $L$ of saldo.

The greatest value of the correlation coefficient is at lag $L=6, s l=0.00319$. Should we already assume that changes in the rate after 6 days lead to a change in liquidity or is it a false correlation? We shall carry out the verification of causality according to Granger [5]. To do this, we estimate the corresponding regression models. Results 


$$
\begin{aligned}
& \hat{M}_{(s l)}^{t}=\underset{(0.0 \ldots)}{49.7}+\underset{(0.0 \ldots)}{0.59} M^{t-6}+\underset{(0.0016)}{35.5} \Delta S^{t-6} \\
& R^{2}=0.267, s l_{\text {tot }}=0.0 \ldots \\
& \underset{(s l)}{\Delta \hat{S}^{t}}=\underset{(0.65)}{0.008}+\underset{(0.584)}{0.016 \Delta S^{t-6}}+\underset{(0.33)}{0.0001} M^{t-6} ; \\
& R^{2}=0.001, s l_{\text {tot }}=0.532
\end{aligned}
$$

Thus, the changes in the rate 6 days ago is the cause (according to Granger) for saldo values (and not vice versa!). If we now consider the estimate $\hat{\rho}\left(M^{t-L}, \Delta S^{t}\right)$ when the saldo precedes the change in the rate, then the largest value of the correlation coefficient is at $\operatorname{lag} L=10, s l=0.056$. An analysis similar to (1) and (2) does not give reason for considering the saldo as the cause for the change in the rate with lag 10 (and vice versa).

\section{Model and Kalman filter}

Observed output value at moments

$$
t=L+1, L+2, \ldots, 1201
$$

is saldo $y^{t}=M^{t}=y 0^{t}+v^{t}$. For the control value at the same moments, in accordance with the analysis of the previous section, we choose preceded $L$ days earlier dollar exchange rate changes $u^{t}=\Delta S^{t-L}$. Renumber the time points (3) as $t=0,1, \ldots, 1201-L-1=1195=T-1$ is the last (current) day of 28.04.2018, on which the saldo and the rate gain are known. We will assume that the "true" value of the saldo $y 0^{t}$ obeys the equation of a discrete oscillator [6] with control and noise

$$
y 0^{t+1}=y 0^{t}-\beta_{0}\left(y 0^{t}-y 0^{t-1}\right)-\beta_{1}\left(y 0^{t-1}-y 0^{t-2}\right)+\beta_{2}\left(u^{t}-u^{t-1}\right)+w_{0}{ }^{t} .
$$

Here $\beta_{0}, \beta_{1}$ - the parameters of the oscillator, fluctuations occur around the previous value; $\beta_{2}$ - parameter of control operator. The oscillatory model often used in the economics [7], is taken from the nature of commercial banks' actions to create money supply: loan - lend - loan payment (for this, again, loan), etc. The type of control term is chosen according to the identified influence $u^{t}$ on $y 0^{t+1}$ and $u^{t-1}$ on $y 0^{t}$, it is clear that the lag $L$ should be taken equal to 5 .

Introducing the state vector $x^{t}=\left(\begin{array}{llll}y 0^{t} & y 0^{t-1} \quad y 0^{t-2} \quad u^{t-1}\end{array}\right)^{T}$, we obtain the evolution $F^{t}=\left(\begin{array}{cccc}1-\beta_{0} & \beta_{0}-\beta_{1} & \beta_{1} & -\beta_{2} \\ 1 & 0 & 0 & 0 \\ 0 & 1 & 0 & 0 \\ 0 & 0 & 0 & 0\end{array}\right)$, control $G^{t}=\left(\begin{array}{llll}\beta_{2} & 0 & 0 & 1\end{array}\right)^{T}$, and observation $H^{t}=\left(\begin{array}{llll}1 & 0 & 0 & 0\end{array}\right)$ operators + in the model of the system in the states space

$$
\begin{gathered}
x^{t+1}=F^{t} x^{t}+G^{t} u^{t}+w^{t}, \\
y^{t}=H^{t} x^{t}+v^{t},
\end{gathered}
$$

where $w^{t}=\left(\begin{array}{llll}w_{0}{ }^{t} & 0 & 0 & 0\end{array}\right)^{T}, \quad v^{t}$ - system and observation noises, $M w^{t}=0, \quad M v^{t}=0, \quad Q=M w^{t} w^{t T}$, $Q_{i j}=\left\{\begin{array}{l}\sigma_{w}^{2}, i=j=0 \\ 0, \text { otherwise }\end{array}, R=M v^{t} v^{t T}=\sigma_{v}^{2}\right.$. The initial moment of the filter work is $T l f=3$, the estimate of the initial state $\hat{x}^{T l f-1 \mid T l f-1}$ is chosen $\left(\begin{array}{llll}y^{T l f-1} & y^{T l f-2} & y^{T l f-3} & u^{T l f-2}\end{array}\right)^{T}$, and the covariance matrix of this estimate $\sigma_{P}^{2} I, I$ is the identity matrix.

Estimation of the model parameters vector

$$
\theta=\left(\sigma_{v} \sigma_{w} \sigma_{P} \beta_{0} \beta_{1} \beta_{2}\right)^{T}
$$

was carried out by minimizing the root-mean-square error of KF prediction of the saldo $\operatorname{RMSE}(\theta)=\sqrt{\frac{1}{T u-(T l-1)} \sum_{t=T l}^{T u}\left(y^{t+1}-\hat{y}^{t+1 \mid t}\right)^{2}}, \hat{\theta}_{R M S E}=\underset{\hat{\theta}}{\arg \min } \operatorname{RMSE}(\hat{\theta})$.

Here $\hat{y}^{t+1 \mid t}$ is the forecast for saldo in moment $t+1$ by information up to the moment $t$. $T l$ is the initial moment, $T u$ is the final moment of the training (fitting) window. Then, using the estimated parameters $\hat{\theta}_{R M S E}$, starting from 
the last estimate of the state, its covariance matrix and $u^{t}$, all at the moment $T u$, we predict by KF (using $y^{t}$ and $\left.u^{t}\right)$ at times $t$ from $T u+1$ to $T-1$ one day ahead $\hat{y}^{t+1 \mid t}$.

The adequacy of the estimated model means its ability to make reasonable predictions beyond the limits of the data used (out-of-sample). The standard error of adequacy characterizes the average error of these forecasts $R M S E A=\sqrt{\frac{1}{T-2-T u} \sum_{t=T u+1}^{T-2}\left(y^{t+1}-\hat{y}^{t+1 \mid t}\right)^{2}}$ (the forecast at time $T-1$ for time $T$ has nothing to compare with, it is the purpose of the calculation).

The estimated model parameters $\hat{\theta}_{R M S E}$ can be used to build the system's transfer matrix $W(z)=H(z I-F)^{-1} G$, its amplitude-frequency characteristic $A F C(\omega)=\left|W\left(e^{i \omega}\right)\right|$, and to obtain a z-transform of the output sequence (saldo) $Y_{Z}(z)$ from the $z$-transform of controls (dollar exchange rate) $U_{Z}(z)$ and the initial state $x^{0}$ $Y_{Z}(z)=W(z) U_{Z}(z)+z H(z I-F)^{-1} x^{0}$. After the inversed z-transformation, we'll get $y W^{t}$ - what it would be if the saldo behaves only under the influence of the existing changes in the dollar exchange rate.

The calculations were carried out in PTC Mathcad Prime.

\section{Interpretation of results}

If you teach (estimate) over all data for 3.5 years from $T l=2$ till $T u=T-2-20$ (except the last 20 points reserved for checking the adequacy), then the estimated parameters (4) are 0.017, 0.016, 12.752, $-0.075,0.211,24.442$. The resulting estimates of oscillator parameters mean that the period of the saldo self-oscillations $2 \pi / \arccos \left(-\hat{\beta}_{0} / 2 \sqrt{\hat{\beta}_{1}}\right)=4.218$ days and the attenuation of the amplitude per day by $1 / \sqrt{\hat{\beta}_{1}}=2.179$ times. $\hat{\beta}_{2}=$ 24.442 means that with the rate growth in 1 RUB, after 6 days the money supply increases by about 20 billion rubles. The achieved RMSE $=165.204$ billion RUB. RMSEA was equal to 161.8 billion RUB. For the random walk model (when the prediction equal to the previous value) for the same 20 points, the adequacy error is RMSEARW $=184.7$ billion RUB. $y W^{t}$, found for the moments after the training, is noticeably different from the KF predictions (which influenced by previous value of the output), it means that there exist jumps in saldo not due to the dollar rate.

In practical forecasting, it is natural to attach to the available data of the last period of time. Therefore, we will conduct training from $T l=1150$ (mid-March 2018) till $T u=T-2-5=1189$ (end of April 2018). The results in Fig.3.

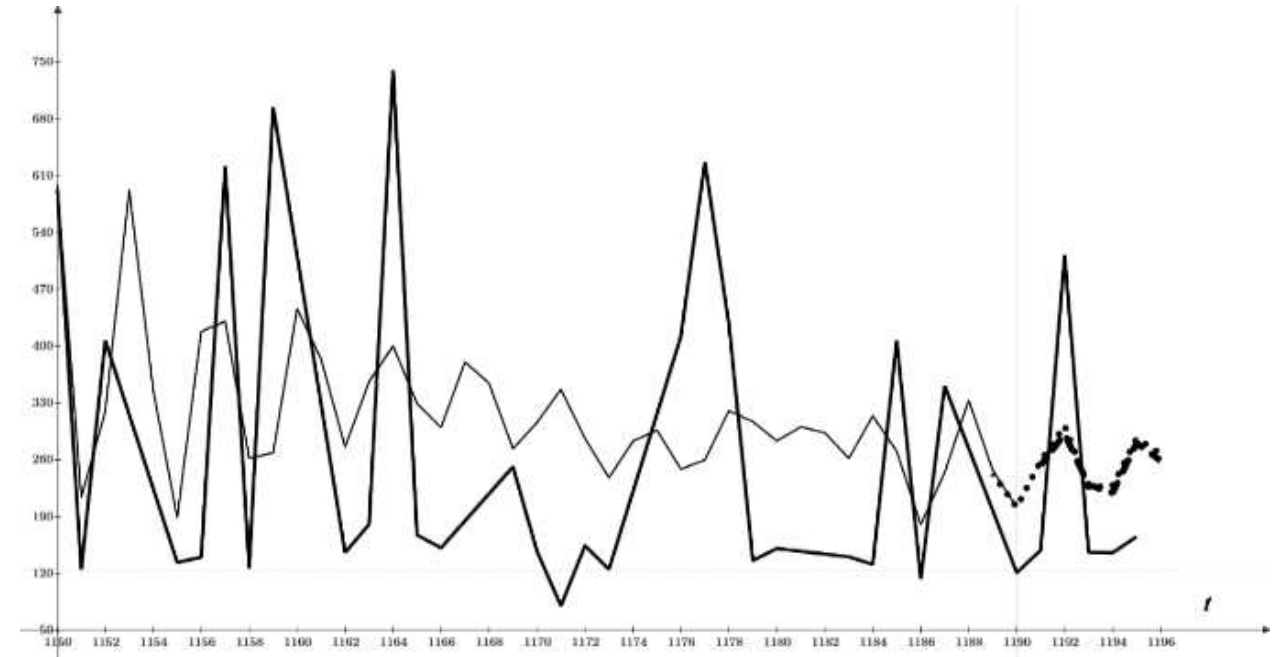

Figure 3. Daily data from 14.03.2018 to 29.04.2018. The observed saldo (solid curve), the forecast fitted to it based on information a day ago (the thin solid curve - training), the forecast by estimated model (dashed curve), $y W^{t}$ - dotted curve.

The estimated parameters (4) are $16.187,2.265,3.165,0.27,0.83,15.868$. The self-oscillations period is 3.654 days (angular frequency $\omega=1.72$ ), the attenuation per day is 1.098 times. In this case, RMSEA $=130.3$ billion RUB, the forecast for saldo in the future moment of 29.04.2018 by estimated model is equal to 259.38 billion RUB. $y W^{t}$ are close to the forecasts by KF, which reflects the leading role of changes in exchange rate in the saldo behavior during this period. The great value (16.187 billion RUB) of the standard deviation of the observation noise and a comparison of the forecast using the estimated model with the actual saldo (see the last 5 days in Fig. 3) show that the latter fluctuations are amplified by some mechanism synchronous with the influence of the 
dollar/ruble rate change.

$A F C$ as a function of the period of input oscillations $\tau=\frac{2 \pi}{\omega}$, has the form (see Fig. 4).

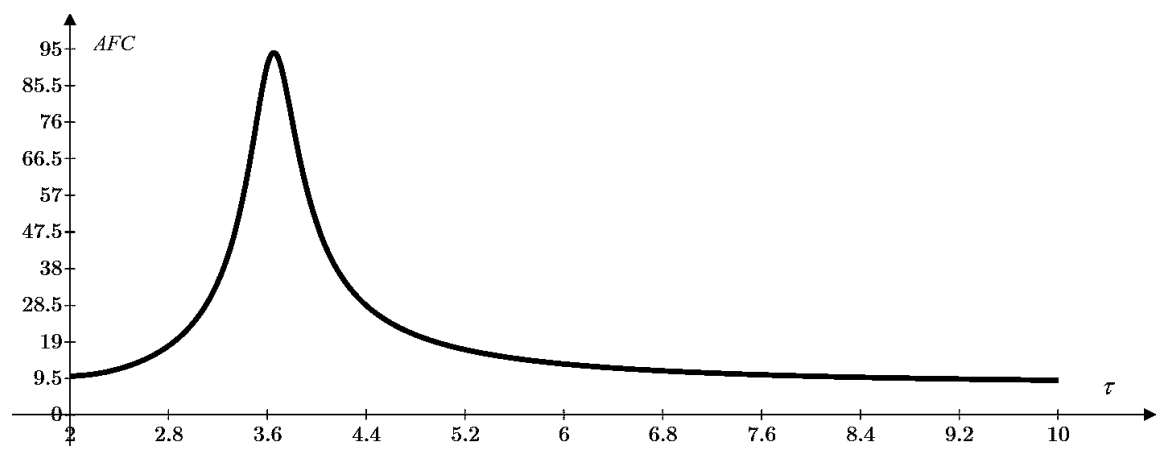

Figure 4. Amplitude-frequency characteristic of the system "change in the dollar rate-liquidity saldo" as a function of the period $\tau$ (days) of rate changes oscillations.

It can be seen that the system of "change in the dollar rate-liquidity saldo" during this period has a high "quality factor", i.e. control $u^{t}$ (changes in the dollar rate), repeated with a period close to $\tau=3.654$, can cause fluctuations in the saldo of large amplitude. In fact, the periodogram estimate of the power spectral density of the control over all data for 3.5 years has a main peak with a period of about 7 days. There is also a slight peak with a period of about 31 days.

\section{Conclusion}

The model proposed in this work affects only one fragment of the extensive system of country's money supply dynamics. In a crisis-free period, it can be used to predict to some extent changes in the ruble money supply by tracking changes in the dollar exchange rate. The possibility of unfavorable swaying of the oscillations of the former was discovered if a period of exchange rate changes would be close to the resonant one.

\section{References}

1. Ozbek L. and Umit Ozale (2005), Employing the Extended Kalman Filter in Measuring the Output Gap, Journal of Economic Dynamics and Control, 29, 1611-22.

2. Borodachev S.M. (2018), "GDP and efficiency of Russian economy", AIP Conference Proceedings 1926, 020011 (2018); https://doi.org/10.1063/1.5020460.

3. Sergei Borodachev. Monthly Forecasting of the Dollar to the Ruble Exchange Rate with Adaptive Kalman Filter. International Journal of Systems Science and Applied Mathematics. Vol. 3, No. 2, 2018, pp. 24-29. doi: 10.11648/j.ijssam.20180302.12

4. Engel C. and Wu S. P. Y. (2018), Liquidity and Exchange Rates: An Empirical Investigation. NBER Working Paper No. 25397, http://www.nber.org/papers/w25397.

5. Granger C. W. J. (1969). "Investigating Causal Relations by Econometric Models and Cross-spectral Methods". Econometrica. 37 (3): 424-438. doi:10.2307/1912791. JSTOR 1912791.

6. Oppenheim A.V., Schafer R.W. Discrete-Time Signal Processing. 3rd edition. — Prentice Hal, 2009.

7. Nonlinear Dynamics in Economics and Social Sciences. Proceedings of the Second Informal Workshop, Held at the Certosa di Pontignano, Siena, Italy, May 27-30, 1991. Eds: Franco Gori Lucio Geronazzo Marcello Galeotti. in Lecture Notes in Economics and Mathematical Systems (1993). 